\title{
Optimality conditions for invex nonsmooth optimization problems with fuzzy objective functions
}

\author{
Tadeusz Antczak ${ }^{1}$ (D)
}

Accepted: 27 December 2021 / Published online: 22 February 2022

(c) The Author(s) 2022

\begin{abstract}
In this paper, the definitions of Clarke generalized directional $\alpha$-derivative and Clarke generalized gradient are introduced for a locally Lipschitz fuzzy function. Further, a nonconvex nonsmooth optimization problem with fuzzy objective function and both inequality and equality constraints is considered. The Karush-Kuhn-Tucker optimality conditions are established for such a nonsmooth extremum problem. For proving these conditions, the approach is used in which, for the considered nonsmooth fuzzy optimization problem, its associated bi-objective optimization problem is constructed. The bi-objective optimization problem is solved by its associated scalarized problem constructed in the weighting method. Then, under invexity hypotheses, (weakly) nondominated solutions in the considered nonsmooth fuzzy minimization problem are characterized through Pareto solutions in its associated bi-objective optimization problem and Karush-Kuhn-Tucker points of the weighting problem.
\end{abstract}

Keywords Nonsmooth optimization problem with fuzzy objective function · Karush-Kuhn-Tucker optimality conditions · Nondominated solution · Weighting method · Invex fuzzy function

\section{Introduction}

In optimization theory, we are used to the fact that, in general, operations research problems are usually modeling as deterministic optimization problems. Whereas, in most real-world applications, optimization problems, which are models of real-world processes, contain uncertain data. The data are often uncertain in models real-world processes, for example, due to estimation errors, prediction errors, or lack of information (for example, optimization problems arising in engineering, industry or commerce might involve various costs, financial returns, and future demands that might be

Tadeusz Antczak

tadeusz.antczak@wmii.uni.lodz.pl

1 Faculty of Mathematics and Computer Science, University of Łódź, Banacha 22, 90-238 Łódź, Poland 
unknown at the time of the decision). There exist various approaches to such optimization problems. This follows from the fact that the methodology for solving uncertain optimization problems has widely applied to many research fields.

One of such approaches used for solving extremum problems with uncertainty data is fuzzy optimization. Tanaka et al. (1984) were first who proposed the concept of fuzzy optimization on general level. With the development of theories of fuzzy numbers and their applications, the concept of fuzzy numbers became more and more important. Since then, fuzzy mathematical programming problems have been extensively studied by many authors. The definition of a convex fuzzy mapping was introduced by Nanda and Kar (1992). Henceforward, the research of convexity for fuzzy mapping and application to convex fuzzy optimization have been developed widely by many authors (see, for example, Ammar and Metz 1992; Chalco-Cano et al. 2016; Panigrahi et al. 2008; Wang and Wu 2003; Yan and Xu 2002, and others). Although convexity notion plays a key role in fuzzy optimization theory, however, this concept is very strict and, therefore, the area of its applications is limited. Recently, therefore, different types of generalized convexity notions for fuzzy mappings have been defined and used also for proving optimality and duality results for various classes of nonconvex fuzzy optimization problems (see, for example, Behera and Nayak 2014; Arana-Jiménez et al. 2015; Mishra et al. 2006; Noor 1994; Osuna-Gómez et al. 2016; Rufián-Lizana et al. 2012; Syau 1999, 2000, 2001, and others).

Although a lot of interesting explorations have been made in the study of optimality conditions for fuzzy optimization problems from different viewpoints, it seems that not much progress has been made in the aspect of proving optimality conditions for such nondifferentiable extremum problems. Subsequently, Wang and Wu (2003) proposed the new concepts of directional derivative, differential and subdifferential of a fuzzy function, and discussed the characterizations of directional derivative and differential of a fuzzy function by using the directional derivative and the differential of two crisp functions that are determined. By using $\alpha$-cut, Ruziyeva and Dempe (2015) derived necessary and sufficient optimality conditions for an optimal solution of the considered nondifferentiable convex fuzzy optimization problem for which they defined its associated bicriterial optimization problem. Then, they showed that a solution of the nondifferentiable fuzzy optimization problem is Pareto optimal for its associated bicriterial optimization problem.

Motivated by the recent work going on in these fields, we discuss several optimality conditions for the considered nondifferentiable fuzzy optimization problem with both inequality and equality constraints in which the involved functions are locally Lipschitz. Therefore, we introduce the definitions of Clarke generalized directional $\alpha$-derivative and Clarke generalized gradient of a locally Lipschitz fuzzy mapping. By using them, we also define the concept of nondifferentiable invexity for a locally Lipschitz fuzzy function. In order to derive optimality conditions for the considered nonsmooth optimization problem with fuzzy objective function and with both inequality and equality constraints, we define its associated bi-objective optimization problem for fixed $\alpha$-cut. Then we characterize the set of (weakly) nondominated solutions in the considered nondifferentiable fuzzy optimization problem through the set of Pareto solutions in its associated bi-objective optimization problem. In order to solve the biobjective optimization problem, we apply the scalarization method. In this approach, 
for the bicriterial optimization problem, we construct the scalarized extremum problem which is the weighting scalar optimization problem. We use Karush-Kuhn-Tucker points of the weighting problem for characterizing optimality the considered nondifferentiable fuzzy optimization problem. This idea allows us to derive the optimality conditions for (weakly) nondominated solutions of the considered nondifferentiable fuzzy optimization problem with both inequality and equality constraints in the form of Karush-Kuhn-Tucker optimality conditions. The results are illustrated by examples of an nondifferentiable invex fuzzy function and a nonsmooth optimization problem with fuzzy objective function in which the involved functions are locally Lipschitz invex (with respect to the same function $\eta$ ). Thus, the optimality conditions established in the paper generalize the similar results previously proved in the literature for differentiable and convex fuzzy optimization problems (see, for example, Chalco-Cano et al. 2016; Panigrahi et al. 2008; Pathak and Pirzada 2001; Ruziyeva and Dempe 2015; Wu 2007), Wu 2008).

\section{Notations and preliminaries}

We first quote some preliminary notations, definitions and results which will be needed in the sequel. Throughout this paper, $R$ is the set of all real numbers is equipped with the usual topology. We denote by $\mathcal{K}_{C}$ the family of all bounded closed intervals in $R$, that is, $\mathcal{K}_{C}=\left\{\left[a^{L}, a^{R}\right]: a^{L}, a^{R} \in R\right.$ and $\left.a^{L} \leq a^{R}\right\}$.

A fuzzy subset of $R^{n}$ is a mapping $\tilde{u}: R^{n} \rightarrow[0,1]$. For each fuzzy set $\tilde{u}$, we denote its $\alpha$-level set as $[\widetilde{u}]^{\alpha}=\left\{x \in R^{n}: \widetilde{u}(x) \geq \alpha\right\}$ for any $\alpha \in(0,1]$. By supp $(\widetilde{u})$, we denote the support of $[\tilde{u}]^{\alpha}$, i.e. the set $\left\{x \in R^{n}: \tilde{u}(x)>0\right\}$. By $\tilde{u}_{0}$ we define the closure of supp $(\widetilde{u})$, i.e. the 0 -level set of $\tilde{u}$.

Definition 1 (Osuna-Gómez et al. 2016) A compact and convex fuzzy set $\tilde{u}$ on $R^{n}$ is a fuzzy set with the following requirements:

(1) $\tilde{u}$ is normal, i.e. there exists $x^{*} \in R^{n}$ such that $\tilde{u}\left(x^{*}\right)=1$,

(2) $\tilde{u}$ is an upper semicontinuous function,

(3) $\tilde{u}(\lambda x+(1-\lambda) y) \geq \min \{\tilde{u}(x), \tilde{u}(y)\}$ for all $x, y \in R^{n}$ and any $\lambda \in[0,1]$.

(4) the 0-level set, i.e. $\tilde{u}_{0}$, is a compact subset of $R^{n}$.

Let $\mathcal{F}(R)$ denote the family of fuzzy intervals in $R$, i.e. $\mathcal{F}(R)$ denotes the family of compact and convex fuzzy sets on $R$. Then, if $\tilde{u} \in \mathcal{F}(R)$, we say that $\tilde{u}$ is a fuzzy interval. Hence, for any $\tilde{u} \in \mathcal{F}(R)$, we have that $[\tilde{u}]^{\alpha}$ is a nonempty compact and convex subset of $R$ for all $\alpha \in[0,1]$. If a fuzzy interval $\tilde{u}$ is such that $[\tilde{u}]^{1}=\{a\}$ for some $a \in R$, i.e. $[\tilde{u}]^{1}$ is a singleton, then $\tilde{u}$ is a fuzzy number (see, Osuna-Gómez et al. 2016). Also any $a \in R$ can be regarded as a fuzzy number $\tilde{a} \in \mathcal{F}(R)$ defined by $\widetilde{a}(x)=\left\{\begin{array}{l}1 \text { if } x=a, \\ 0 \text { if } x \neq a\end{array}\right.$ In particular, the fuzzy number $\widetilde{\mathcal{0}} \in \mathcal{F}(R)$ is defined as $\widetilde{0}(x)=1$ if $x=0$, and $\widetilde{0}(x)=0$, otherwise.

Thus, the $\alpha$-levels of a fuzzy interval are given by $\left[u^{L}(\alpha), u^{R}(\alpha)\right], u^{L}(\alpha), u^{R}(\alpha)$ $\in R, u^{L}(\alpha) \leq u^{R}(\alpha)$, for all $\alpha \in[0,1]$. 
Remark 2 In Wu (2007), Wu used the notation $\tilde{1}_{\{a\}}$ to represent the crisp number with value $a$. It is easy to see that, one has

$$
\left(\widetilde{1}_{\{a\}}\right)^{L}(\alpha)=\left(\widetilde{1}_{\{a\}}\right)^{R}(\alpha)=a
$$

for all $\alpha \in[0,1]$. Hence, a real number $a$ in $\mathrm{Wu}(2007)$ is regarded as a crisp number $\widetilde{1}_{\{a\}}$ in order to short the form of a real number.

Given fuzzy intervals $\tilde{u}, \tilde{v} \in \mathcal{F}(R)$ represented by $[\tilde{u}]^{\alpha}=\left[u^{L}(\alpha), u^{R}(\alpha)\right]$ and $[\widetilde{v}]^{\alpha}=\left[v^{L}(\alpha), v^{R}(\alpha)\right]$, respectively, and a real number $k$. Then the fuzzy addition $\tilde{u}+\widetilde{v}$ and scalar multiplication $k \tilde{u}$ are defined as follows (see Panigrahi et al. 2008; Rufián-Lizana et al. 2012; Wang and Wu 2003):

(a) $(\tilde{u}+\widetilde{v})(x)=\sup _{y+z=x} \min \{\tilde{u}(y), \widetilde{v}(z)\}$,

(b) $(k \tilde{u})(x)=\left\{\begin{array}{cl}\widetilde{u}\left(\frac{x}{k}\right), & \text { if } k \neq 0, \\ \widetilde{0}, & \text { if } k=0,\end{array}\right.$, where $\widetilde{0} \in \mathcal{F}(R)$.

The above operations can be defined in the equivalent way. Namely, for every $\alpha \in[0,1]$, we have:

$$
[\tilde{u}+\widetilde{v}]^{\alpha}=\left[(u+v)^{L}(\alpha),(u+v)^{R}(\alpha)\right]=\left[u^{L}(\alpha)+v^{L}(\alpha), u^{R}(\alpha)+v^{R}(\alpha)\right]
$$

and

$$
\begin{aligned}
{[k \tilde{u}]^{\alpha} } & =\left[(k u)^{L}(\alpha),(k u)^{R}(\alpha)\right] \\
& =\left[\min \left\{k u^{L}(\alpha), k u^{R}(\alpha)\right\}, \max \left\{k u^{L}(\alpha), k u^{R}(\alpha)\right\}\right] .
\end{aligned}
$$

Definition 3 (Wu 2007) Let $\tilde{u}$ and $\widetilde{v}$ be two fuzzy numbers. The Hukuhara difference ( $H$-difference for short) (denoted by $\widetilde{u} \ominus_{H} \widetilde{v}$ ) is the fuzzy number $\widetilde{w}$, if it exists, such that

$$
\tilde{u} \ominus_{H} \tilde{v}=\widetilde{w} \Longleftrightarrow \widetilde{u}=\widetilde{v}+\widetilde{w} .
$$

Note that addition is commutative and $\widetilde{w}$ is unique.

Proposition 4 (Wu 2007) Let $\tilde{u}$ and $\widetilde{v}$ be two fuzzy numbers. If the Hukuhara difference $\widetilde{w}=\tilde{u} \ominus_{H} \tilde{v}$ exists, then $\widetilde{w}^{L}(\alpha)=u^{L}(\alpha)-v^{L}(\alpha)$ and $\widetilde{w}^{R}(\alpha)=u^{R}(\alpha)-v^{R}(\alpha)$ for each $\alpha \in[0,1]$.

Definition 5 A triangular fuzzy number $\tilde{u}$ is a fuzzy number represented with three points $\tilde{u}=\left(u_{1}, u_{2}, u_{3}\right)$. Its membership function is defined by

$$
\tilde{u}(x)= \begin{cases}\frac{x-u_{1}}{u_{2}-u_{1}} & \text { if } u_{1} \leq x \leq u_{2} \\ \frac{u_{3}-x}{u_{3}-u_{2}} & \text { if } u_{2} \leq x \leq u_{3} \\ 0 & \text { otherwise }\end{cases}
$$


The $\alpha$-level set of a triangular fuzzy number is defined by

$$
[\widetilde{u}]^{\alpha}=\left[u^{L}(\alpha), u^{R}(\alpha)\right]=\left[(1-\alpha) u_{1}+\alpha u_{2},(1-\alpha) u_{3}+\alpha u_{2}\right]
$$

In order to compare two fuzzy numbers, in the recent literature, there are various definitions as a generalization of the relationship on intervals. In the paper, we shall use the concepts of partial orderings proposed by (Wu 2008), which are similar to the similar concepts used for multiobjective programming problems.

Let $\tilde{u}, \tilde{v} \in \mathcal{F}(R)$ be two fuzzy numbers represented by $[\tilde{u}]^{\alpha}=\left[u^{L}(\alpha), u^{R}(\alpha)\right]$ and $[\widetilde{v}]^{\alpha}=\left[v^{L}(\alpha), v^{R}(\alpha)\right]$, respectively.

Definition 6 (Wu 2008) We say that $\tilde{u}$ dominates (is better than) $\widetilde{v}$ if and only if $[\widetilde{u}]^{\alpha} \preceq[\widetilde{v}]^{\alpha}$ for all $\alpha \in[0,1]$. In other words, $\tilde{u}$ dominates (is better than) $\widetilde{v}$ if and only if

$$
\left\{\begin{array} { l } 
{ u ^ { L } ( \alpha ) < v ^ { L } ( \alpha ) } \\
{ u ^ { R } ( \alpha ) \leq v ^ { R } ( \alpha ) }
\end{array} \text { or } \left\{\begin{array} { l } 
{ u ^ { L } ( \alpha ) \leq v ^ { L } ( \alpha ) } \\
{ u ^ { R } ( \alpha ) < v ^ { R } ( \alpha ) }
\end{array} \text { or } \left\{\begin{array}{l}
u^{L}(\alpha)<v^{L}(\alpha) \\
u^{R}(\alpha)<v^{R}(\alpha)
\end{array} \text { for all } \alpha \in[0,1]\right.\right.\right.
$$

Definition 7 (Wu 2008) We say that $\tilde{u}$ strongly dominates $\widetilde{v}$ if and only if $[\tilde{u}]^{\alpha} \prec[\widetilde{v}]^{\alpha}$ for all $\alpha \in[0,1]$. In other words, $\tilde{u}$ dominates (is better than) $\widetilde{v}$ if and only if

$$
\begin{gathered}
\left\{\begin{array}{l}
u^{L}(\alpha)<v^{L}(\alpha) \\
u^{R}(\alpha) \leq v^{R}(\alpha)
\end{array} \text { for all } \alpha \in[0,1]\right. \\
\text { or }\left\{\begin{array}{l}
u^{L}(\alpha) \leq v^{L}(\alpha) \\
u^{R}(\alpha)<v^{R}(\alpha)
\end{array} \text { for all } \alpha \in[0,1]\right. \\
\text { or }\left\{\begin{array}{l}
u^{L}(\alpha)<v^{L}(\alpha) \\
u^{R}(\alpha)<v^{R}(\alpha)
\end{array} \text { for all } \alpha \in[0,1] .\right.
\end{gathered}
$$

Remark 8 (Wu 2007, 2008) It is not difficult to see that if $\widetilde{u}$ strongly dominates $\widetilde{v}$, then $\tilde{u}$ dominates $\widetilde{v}$.

\section{Locally Lipschitz fuzzy functions}

At the beginning this section, we re-call some well-known definitions and notations for nondifferentiable real-valued crisp functions.

A crisp function $f: R^{n} \rightarrow R$ is locally Lipschitz at a point $\widehat{x} \in R^{n}$ if there exist scalars $K_{\widehat{x}}>0$ and $\varepsilon>0$ such that, the inequality $|f(y)-f(z)| \leqq K_{\hat{x}}\|y-z\|$ holds for all $y, z \in \widehat{x}+\varepsilon B$, where $B$ signifies the open unit ball in $R^{n}$, so that $\widehat{x}+\varepsilon B$ is the open ball of radius $\varepsilon$ about $\widehat{x}$. It is said that $f$ is locally Lipschitz (on $R^{n}$ ) if it is locally Lipschitz at each point of $R^{n}$.

Definition 9 (Clarke 1983) The Clarke generalized directional derivative of a locally Lipschitz function $f: R^{n} \rightarrow R$ at $x \in R^{n}$ in the direction $d \in R^{n}$, denoted by 
$f^{0}(x ; d)$, is given by

$$
f^{0}(x ; d)=\limsup _{\substack{y \rightarrow x \\ \lambda \downarrow 0}} \frac{f(y+\lambda d)-f(y)}{\lambda} .
$$

Definition 10 (Clarke 1983) The Clarke generalized subgradient of the crisp function $f: R^{n} \rightarrow R$ at $x \in R^{n}$, denoted by $\partial f(x)$, is defined as follows:

$$
\partial f(x)=\left\{\xi \in R^{n}: f^{0}(x ; d) \geq \xi^{T} d \text { for all } d \in R^{n}\right\} .
$$

It follows that, for any $d \in R^{n}, f^{0}(x ; d)=\max \left\{\xi^{T} d: \xi \in \partial f(x)\right\}$.

Lemma 11 (Clarke 1983) Let $f: X \rightarrow R$ be a locally Lipschitz function on $a$ nonempty open set $X \subset R^{n}, x$ is an arbitrary point of $X$ and $\lambda \in R$. Then

$$
\partial(\lambda f)(x) \subseteq \lambda \partial f(x)
$$

Proposition 12 (Clarke 1983) Let $f_{i}: X \rightarrow R, i=1, \ldots, k$, be crisp convex functions on a nonempty open set $X \subset R^{n}, x$ is an arbitrary point of $X \subset R^{n}$. Then

$$
\partial\left(\sum_{i=1}^{k} f_{i}\right)(x) \subseteq \sum_{i=1}^{k} \partial f_{i}(x)
$$

Equality holds in the above relation if all but at most one of the functions $f_{i}$ are strictly differentiable at $u$.

Corollary 13 (Clarke 1983) For any scalars $\lambda_{i}$, one has

$$
\partial\left(\sum_{i=1}^{k} \lambda_{i} f_{i}\right)(x) \subseteq \sum_{i=1}^{k} \lambda_{i} \partial f_{i}(x)
$$

and equality holds if all but at most one of the $f_{i}$ is strictly differentiable at $x$.

Remark 14 If each $f_{i}$ is a crisp convex function at $x$, equality holds in Proposition 12. Equality then holds in Corollary 13 as well, if in addition each $\lambda_{i}$ is nonnegative.

The concept of nondifferentiable invexity for locally Lipschitz functions was defined by Reiland (1990).

Definition 15 Let $f: R^{n} \rightarrow R$ be a locally Lipschitz crisp function and $\widehat{x} \in R^{n}$ be given. If there exists a vector-valued function $\eta: R^{n} \times R^{n} \rightarrow R^{n}$ such that the following inequality

$$
f(x)-f(\widehat{x}) \geq \xi^{T} \eta(x, \widehat{x}), \forall \xi \in \partial f(\widehat{x}) \quad(>)
$$


holds for all $x \in R^{n},(x \neq \widehat{x})$, then it is said that $f$ is a (strictly) invex function at $\widehat{x}$ on $R^{n}$. If (6) is satisfied at any point $\widehat{x}$, then $\widetilde{f}$ is a (strictly) invex function on $R^{n}$. If (6) is satisfied on a nonempty subset $X \subset R^{n}$, then $f$ is a (strictly) invex function on $X$.

Proposition 16 (Clarke 1983) Let the crisp function $f: R^{n} \rightarrow R$ be locally Lipschitz. at a point $\widehat{x} \in R^{n}$ and attain its (local) minimum at $\widehat{x}$. Then $0 \in \partial f(\widehat{x})$.

Now, we give the definition of a fuzzy mapping given, for example, by Panigrahi et al. (2008).

Definition 17 (Panigrahi et al. 2008) Let $X$ be a nonempty subset of $R^{n}$. Then $\tilde{f}$ : $X \rightarrow \mathcal{F}(R)$ is said to be a fuzzy mapping. We associate with $\widetilde{f}$ the family of intervalvalued functions $\widetilde{f}_{\alpha}: X \rightarrow \mathcal{K}_{C}$ given by $\widetilde{f}_{\alpha}(x)=[\widetilde{f}(x)]^{\alpha}, \alpha \in[0,1]$. The $\alpha$-cut of $\widetilde{f}$ at $x \in X$, which is a closed and bounded interval for each $\alpha \in[0,1]$, can be denoted by

$$
\widetilde{f}_{\alpha}(x)=\left[f^{L}(x, \alpha), f^{R}(x, \alpha)\right],
$$

where $f^{L}(x, \alpha)=\min \widetilde{f}_{\alpha}(x)$ and $f^{R}(x, \alpha)=\max \tilde{f}_{\alpha}(x)$. Thus, $\tilde{f}$ can be understood by two functions $f^{L}$ and $f^{R}$, which are functions from $X \times[0,1]$ to the set $R, f^{L}$ is a bounded increasing function of $\alpha, f^{R}$ is a bounded decreasing function of $\alpha$ and, moreover, $f^{L}(x, \alpha) \leq f^{R}(x, \alpha)$ for all $x \in X$ and each $\alpha \in[0,1]$. Here, the endpoint functions $f^{L}, f^{R}: X \times[0,1] \rightarrow R$ are called left- and right-hand side functions of $\widetilde{f}_{\alpha}(\cdot)$, respectively.

It is known that continuity and differentiablity of a fuzzy function $\tilde{f}$ can be defined through continuity and differentiablity of its left- and right side functions for fixed $\alpha$. Namely, the definition of a level-wise differentiable fuzzy function was introduced by Wu (2007) (see also Chalco-Cano et al. 2016; Wu 2009).

Definition 18 (Wu 2007) Let $\tilde{f}: X \rightarrow \mathcal{F}(R)$ be a fuzzy function defined on an open subset $X$ of $R^{n}$ and $\widehat{x} \in X$ be fixed. We say that the fuzzy function $\widetilde{f}$ is level-wise differentiable at $\widehat{x}$ if and only if the real-valued functions $f^{L}(\cdot, \alpha)$ and $f^{R}(\cdot, \alpha)$ are differentiable at $\widehat{x}$ for all $\alpha \in[0,1]$ (which imply that all partial derivatives $\partial f^{L}(\cdot, \alpha) / \partial x_{i}$ and $\partial f^{R}(\cdot, \alpha) / \partial x_{i}, i=1, \ldots, n$, exist at $\widehat{x}$ for all $\alpha \in[0,1])$. We denote derivatives of $f^{L}(\cdot, \alpha)$ and $f^{R}(\cdot, \alpha)$ by $\left(f^{L}\right)^{\prime}(\cdot, \alpha)$ and $\left(f^{R}\right)^{\prime}(\cdot, \alpha)$, respectively. Then, the derivative of $\tilde{f}$ at $\hat{x} \in X$ for the fixed $\alpha \in[0,1]$ is defined as a pair of derivatives $\left(f^{L}\right)^{\prime}(\widehat{x}, \alpha)$ and $\left(f^{R}\right)^{\prime}(\widehat{x}, \alpha)$, that is, $(\widetilde{f})_{\alpha}^{\prime}(\widehat{x})=\left(\left(f^{L}\right)^{\prime}(\widehat{x}, \alpha),\left(f^{R}\right)^{\prime}(\widehat{x}, \alpha)\right)$.

Now, in a natural way, we extend the well-known definition of a locally Lipschitz function to a fuzzy mapping.

Definition 19 It is said that a fuzzy mapping $\tilde{f}: R^{n} \rightarrow \mathcal{F}(R)$ is a locally Lipschitz function at a point $\widehat{x} \in R^{n}$ if the functions $f^{L}(\cdot, \alpha)$ and $f^{R}(\cdot, \alpha)$ are locally Lipschitz at $\widehat{x}$ for each $\alpha \in[0,1]$. 
Now, we introduce the definition of the Clarke generalized derivative of a locally Lipschitz fuzzy function as a pair of functions which need not to be an interval. Namely, for the fixed $\alpha$-cut of the locally Lipschitz fuzzy function $\tilde{f}$, we define the Clarke generalized derivative at $\widehat{x} \in X$ as a pair of Clarke generalized derivatives at $\widehat{x} \in X$ of the functions $f^{L}(\cdot, \alpha)$ and $f^{R}(\cdot, \alpha)$.

Definition 20 The Clarke generalized directional $\alpha$-derivative of the locally Lipschitz fuzzy function $\widetilde{f}$ (given by (2)) at $\widehat{x}$ for some $\alpha$-cut $\widetilde{f}_{\alpha}$ in the direction $d$ is defined as the pair of the Clarke generalized directional $\alpha$-derivatives of the functions $f^{L}(\cdot, \alpha)$ and $f^{R}(\cdot, \alpha)$ at $\widehat{x}$ in the direction $d$ as follows

$$
\begin{aligned}
& \widetilde{f}_{\alpha}^{0}(\widehat{x} ; d) \\
& :=\left(\limsup _{\substack{y \rightarrow \widehat{x} \\
t \downarrow 0}} \frac{f^{L}(y+t d, \alpha)-f^{L}(y, \alpha)}{t}, \limsup _{\substack{y \rightarrow \widehat{x} \\
t \downarrow 0}} \frac{f^{R}(y+t d, \alpha)-f^{R}(y, \alpha)}{t}\right) \\
& \quad:=\left(\left(f^{L}\right)^{0}(\widehat{x}, \alpha ; d),\left(f^{R}\right)^{0}(\widehat{x}, \alpha ; d)\right) .
\end{aligned}
$$

Definition 21 We say that the fuzzy function $\tilde{f}: X \rightarrow \mathcal{F}(R)$ is directionally differentiable in the sense of Clarke at $\widehat{x}$ if $\widetilde{f}_{\alpha}^{0}(\widehat{x} ; d)$ exists for each direction $d$ and for all $\alpha$-cuts.

Now, for the fixed $\alpha$-cut of the locally Lipschitz fuzzy function $\widetilde{f}$, we introduce the definition of its Clarke generalized gradient at the given point as a pair of Clarke generalized gradients at this point of the functions $f^{L}(\cdot, \alpha)$ and $f^{R}(\cdot, \alpha)$.

Definition 22 Let the locally Lipschitz fuzzy function $\tilde{f}: X \rightarrow \mathcal{F}(R)$ admit the Clarke generalized directional $\alpha$-derivative at $\widehat{x}$ in each direction $d \in R^{n}$ for some $\alpha$-cut. The Clarke generalized gradient of $\tilde{f}$ at $\hat{x}$ on the $\alpha$-cut is defined as a pair of Clarke generalized gradients at $\widehat{x}$ of left- and right-hand side functions on this $\alpha$-cut, that is, a pair

$$
\partial \tilde{f}_{\alpha}(\widehat{x}):=\left(\partial f^{L}(\widehat{x}, \alpha), \partial f^{R}(\widehat{x}, \alpha)\right)
$$

where $\partial f^{L}(\widehat{x}, \alpha):=\left\{\xi^{L} \in R^{n}:\left(f^{L}\right)^{0}(\widehat{x}, \alpha ; d) \geq\left\langle\xi^{L}, d\right\rangle\right.$ for all $\left.d \in R^{n}\right\}$ and $\partial f^{R}(\widehat{x}, \alpha):=\left\{\xi^{R} \in R^{n}:\left(f^{R}\right)^{0}(\widehat{x}, \alpha ; d) \geq\left\langle\xi^{R}, d\right\rangle\right.$ for all $\left.d \in R^{n}\right\}$.

We illustrate the definition of the Clarke generalized gradient of a locally Lipschitz fuzzy function by the following example.

Example 23 We define the fuzzy mapping $\widetilde{f}: R \rightarrow \mathcal{F}(R)$ by $\widetilde{f}(x)=\widetilde{2}|x|+\widetilde{1}$, where $\widetilde{1}$ and $\widetilde{2}$ are continuous triangular fuzzy numbers defined as triples $\tilde{1}=(0,1,2)$ and $\widetilde{2}=(0,2,4)$. Then, by (5), it follows that $[\widetilde{1}]^{\alpha}=[\alpha, 2-\alpha]$ and $[\widetilde{2}]^{\alpha}=[2 \alpha, 4-2 \alpha]$. 
Therefore, the $\alpha$-levels of $\tilde{f}$ at $\widehat{x} \in R$ are as follows

$$
\tilde{f}_{\alpha}(\widehat{x})=\left\{\begin{array}{c}
{[-2 \alpha \widehat{x}+\alpha,(2 \alpha-4) \hat{x}+2-\alpha] \text { if } \widehat{x}<0} \\
{[2 \alpha \widehat{x}+\alpha,(4-2 \alpha) \widehat{x}+2-\alpha] \text { if } \widehat{x} \geq 0}
\end{array}\right.
$$

Then,

$$
\begin{aligned}
f^{L}(\widehat{x}, \alpha) & =\left\{\begin{array}{c}
-2 \alpha \widehat{x}+\alpha \text { if } \widehat{x}<0, \\
2 \alpha \widehat{x}+\alpha \text { if } \widehat{x} \geq 0,
\end{array}\right. \\
f^{R}(\widehat{x}, \alpha) & =\left\{\begin{array}{l}
(2 \alpha-4) \widehat{x}+2-\alpha \text { if } \widehat{x}<0, \\
(4-2 \alpha) \widehat{x}+2-\alpha \text { if } \widehat{x} \geq 0 .
\end{array}\right.
\end{aligned}
$$

Clearly, $\tilde{f}$ is a locally Lipschitz fuzzy function on $R$ since the functions $f^{L}(\cdot, \alpha)$ and $f^{R}(\cdot, \alpha)$ are locally Lipschitz on $R$ for each $\alpha \in[0,1]$. Thus, by Definition 22 , the Clarke generalized gradient of $\tilde{f}$ at $\widehat{x}$ is as follows

$$
\partial \widetilde{f}_{\alpha}(\widehat{x})= \begin{cases}(-2 \alpha, 2 \alpha-4) & \text { if } \widehat{x}<0 \\ ([-2 \alpha, 2 \alpha],[2 \alpha-4,4-2 \alpha]) & \text { if } \widehat{x}=0 \\ (2 \alpha, 4-2 \alpha) & \text { if } \widehat{x}>0\end{cases}
$$

Remark 24 It follows by the above definition that, for some $\alpha$-cut and any $d \in R^{n}$, we have

$$
\begin{gathered}
\widetilde{f}_{\alpha}^{0}(\widehat{x} ; d)=\left(\left(f^{L}\right)^{0}(\widehat{x}, \alpha ; d),\left(f^{R}\right)^{0}(\widehat{x}, \alpha ; d)\right)= \\
\left(\max \left\{\left\langle\xi^{L}, d\right\rangle: \xi^{L} \in \partial f^{L}(\widehat{x}, \alpha)\right\}, \max \left\{\left\langle\xi^{R}, d\right\rangle: \xi^{R} \in \partial f^{R}(\widehat{x}, \alpha)\right\}\right) .
\end{gathered}
$$

In Syau (2000), Syau introduced the notion of invexity for a differentiable fuzzy function. Now, using the $\alpha$-cuts for a fuzzy function given by $\mathrm{Wu}(2007,2008)$, we introduce the notion of invexity for a locally Lipschitz fuzzy function via invexity of left- and right-hand side functions $f^{L}(\cdot, \alpha)$ and $f^{R}(\cdot, \alpha)$.

Definition 25 Let $\tilde{f}: R^{n} \rightarrow \mathcal{F}(R)$ be a locally Lipschitz function and $\widehat{x}$ be a given point. If there exists a vector-valued function $\eta: R^{n} \times R^{n} \rightarrow R^{n}$ such that the inequalities

$$
\begin{aligned}
& f^{L}(x, \alpha)-f^{L}(\widehat{x}, \alpha) \geq\left\langle\xi^{L}, \eta(x, \widehat{x})\right\rangle, \forall \xi^{L} \in \partial f^{L}(\widehat{x}, \alpha), \quad(>) \\
& f^{R}(x, \alpha)-f^{R}(\widehat{x}, \alpha) \geq\left\langle\xi^{R}, \eta(x, \widehat{x})\right\rangle, \forall \xi^{R} \in \partial f^{R}(\widehat{x}, \alpha) \quad(>)
\end{aligned}
$$

hold for all $x \in R^{n},(x \neq \widehat{x})$, then it is said that $\tilde{f}$ is a (strictly) invex fuzzy function at $\widehat{x}$ on $R^{n}$. If (8) and (9) are satisfied at any point $\widehat{x}$, then $\widetilde{f}$ is a (strictly) invex fuzzy function on $R^{n}$. If ( 8) and (9) are satisfied on a nonempty subset $X \subset R^{n}$, then $\widetilde{f}$ is a (strictly) invex fuzzy function on $X$. 
In order to illustrate the concept of invexity for fuzzy mappings introduced above, we give an example of such a fuzzy function.

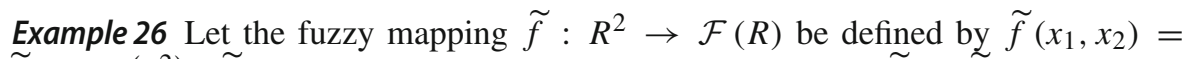
$\widetilde{1} \arctan \left(x_{1}^{2}\right)+\widetilde{3} x_{2}$, where continuous triangular fuzzy numbers $\widetilde{1}$ and $\widetilde{3}$ are defined as triples $\widetilde{1}=(0,1,2)$ and $\widetilde{3}=(1,3,5)$. Then, by (5), the $\alpha$-level set of both triangular fuzzy numbers are defined by $[\tilde{1}]^{\alpha}=[\alpha, 2-\alpha]$ and $[\widetilde{3}]^{\alpha}=[1+2 \alpha, 5-2 \alpha]$, respectively. Further, by (2) and (3), the $\alpha$-level cut of the fuzzy function $\tilde{f}$ is defined as follows

$$
\begin{aligned}
& \tilde{f}_{\alpha}\left(x_{1}, x_{2}\right) \\
& \quad=\left\{\begin{array}{l}
{\left[\alpha \arctan \left(x_{1}^{2}\right)+(1+2 \alpha) x_{2},(2-\alpha) x_{1}^{2}+(5-2 \alpha) x_{2}\right] \text { if } x_{1} \in R, x_{2} \geq 0} \\
{\left[\alpha \arctan \left(x_{1}^{2}\right)+(5-2 \alpha) x_{2},(2-\alpha) x_{1}^{2}+(1+2 \alpha) x_{2}\right] \text { if } x_{1} \in R, x_{2}<0}
\end{array}\right.
\end{aligned}
$$

for all $\alpha \in[0,1]$. Hence, the left- and right-hand side functions $f^{L}(\cdot, \alpha)$ and $f^{R}(\cdot, \alpha)$ are defined by

$$
\begin{aligned}
& f^{L}\left(\left(x_{1}, x_{2}\right), \alpha\right)=\left\{\begin{array}{l}
\alpha \arctan \left(x_{1}^{2}\right)+(1+2 \alpha) x_{2} \text { if } x_{1} \in R, x_{2} \geq 0, \\
\alpha \arctan \left(x_{1}^{2}\right)+(5-2 \alpha) x_{2} \text { if } x_{1} \in R, x_{2}<0,
\end{array}\right. \\
& f^{R}\left(\left(x_{1}, x_{2}\right), \alpha\right)=\left\{\begin{array}{l}
(2-\alpha) \arctan \left(x_{1}^{2}\right)+(5-2 \alpha) x_{2} \text { if } x_{1} \in R, x_{2} \geq 0, \\
(2-\alpha) \arctan \left(x_{1}^{2}\right)+(1+2 \alpha) x_{2} \text { if } x_{1} \in R, x_{2}<0 .
\end{array}\right.
\end{aligned}
$$

Clearly, the left- and right-hand functions $f^{L}(\cdot, \alpha)$ and $f^{R}(\cdot, \alpha)$ are not convex and so $\widetilde{f}$ is not convex. Further, also note that $f^{L}(\cdot, \alpha)$ and $f^{R}(\cdot, \alpha)$ are not differentiable at $\widehat{x}=(0,0)$ and, therefore, $\widetilde{f}$ is not level-wise differentiable at this point (see Definition $4.2 \mathrm{Wu}(2007))$. We now show that $\tilde{f}$ is a locally Lipschitz strictly invex fuzzy function at $\widehat{x}=(0,0)$ on $R^{2}$. In order to do this, let us define $\eta: R^{2} \times R^{2} \rightarrow R^{2}$ by

$$
\eta(x, \widehat{x})=\left\{\begin{array}{l}
{\left[\begin{array}{c}
x_{1}-\widehat{x}_{1} \\
\frac{2-\alpha}{5-2 \alpha}\left(x_{2}-\widehat{x}_{2}\right)
\end{array}\right] \text { if } x_{1} \in R, x_{2} \geq 0,} \\
{\left[\begin{array}{c}
x_{1}-\widehat{x}_{1} \\
\frac{5-2 \alpha}{2-\alpha}\left(x_{2}-\widehat{x}_{2}\right)
\end{array}\right] \text { if } x_{1} \in R, x_{2}<0 .}
\end{array}\right.
$$

Note that, for the functions $f^{L}(\cdot, \alpha)$ and $f^{R}(\cdot, \alpha),(8)$ and (9) are fulfilled as strictly inequalities at $\widehat{x}=(0,0)$ for all $x \in R^{2},(x \neq \widehat{x})$, with respect to $\eta$ defined above. Then, by Definition 25 , the fuzzy objective function $\widetilde{f}$ is strictly invex at $\widehat{x}=(0,0)$ on $R^{2}$ with respect to $\eta$ defined above.

In the paper, we consider fuzzy functions $\tilde{f}: R^{n} \rightarrow \mathcal{F}(R)$ such that their functions $f^{L}(\cdot, \alpha)$ and $f^{R}(\cdot, \alpha)$ are locally Lipschitz at a given point $x$ of interest for each $\alpha \in[0,1]$. 


\section{Fuzzy optimization problem and optimality}

In the present paper, the following optimization problem with fuzzy-valued objective function is considered

$$
\begin{gathered}
\text { minimize } \tilde{f}(x) \\
\text { subject to } \quad g_{j}(x) \leq 0, j \in J=\{1, \ldots m\}, \\
h_{i}(x)=0, i \in I=\{1, \ldots, r\},
\end{gathered}
$$

where the objective function $\tilde{f}: R^{n} \rightarrow \mathcal{F}(R)$ is a fuzzy-valued function, $g_{j}$ : $R^{n} \rightarrow R, j \in J, h_{i}: R^{n} \rightarrow R, i \in I$, are real-valued functions. We call (FOP) the fuzzy optimization problem or the optimization problem with fuzzy objective function. Let $D:=\left\{x \in R^{n}: g_{j}(x) \leq 0, j \in J, h_{i}(x)=0, i \in I\right\}$ be the set of all feasible solutions of the problem (FOP). Further, we denote the set of active inequality constraints at point $\widehat{x} \in D$ by $J(\widehat{x})=\left\{j \in J: g_{j}(\widehat{x})=0\right\}$.

In the present paper, the $\alpha$-cuts are used to describe the objective function, as it was done by $\mathrm{Wu}$ (2007), and it is assumed that its left- and right-hand side values are given by the functions $f^{L}(\cdot, \alpha)$ and $f^{R}(\cdot, \alpha)$ for $\alpha \in[0,1]$, respectively.

Since " $\preceq$ " and " $\prec$ " are partial orderings on $\mathcal{F}(R)$, we may follow for the considered optimization problem with the fuzzy-valued objective function the similar solution concepts used for multiobjective programming problems. Namely, for such optimization problems, we define their optimal solutions as weakly nondominated solutions and nondominated solutions defined by Wu (2008).

Definition 27 (Wu 2008) It is said that a feasible solution $\widehat{x}$ of the considered constrained optimization problem (FOP) with fuzzy-valued objective function is its weakly nondominated solution if there exists no others $x \in D$ such that

$$
\tilde{f}(x) \prec \tilde{f}(\widehat{x}) .
$$

In other words, (by Definition 7), if $\widehat{x} \in D$ is a weakly nondominated solution of the problem (FOP), then there exists no others $x \in D$ such that

$$
\begin{gathered}
\left\{\begin{array}{l}
f^{L}(x, \alpha)<f^{L}(\widehat{x}, \alpha) \\
f^{R}(x, \alpha) \leq f^{R}(\widehat{x}, \alpha)
\end{array} \text { for all } \alpha \in[0,1]\right. \\
\text { or }\left\{\begin{array}{l}
f^{L}(x, \alpha) \leq f^{L}(\widehat{x}, \alpha) \\
f^{R}(x, \alpha)<f^{R}(\widehat{x}, \alpha)
\end{array} \text { for all } \alpha \in[0,1]\right. \\
\text { or }\left\{\begin{array}{l}
f^{L}(x, \alpha)<f^{L}(\widehat{x}, \alpha) \\
f^{R}(x, \alpha)<f^{R}(\widehat{x}, \alpha)
\end{array} \text { for all } \alpha \in[0,1] .\right.
\end{gathered}
$$

Definition 28 (Wu 2008) It is said that a feasible solution $\widehat{x}$ of the considered constrained optimization problem (FOP) with fuzzy-valued objective function is its 
nondominated solution if there exists no others $x \in D$ such that

$$
\widetilde{f}(x) \preceq \widetilde{f}(\widehat{x})
$$

In other words, (by Definition 6), if $\widehat{x} \in D$ is a nondominated solution of the problem (FOP), then there exists no others $x \in D$ such that

$$
\begin{aligned}
& \left\{\begin{array} { l } 
{ f ^ { L } ( x , \alpha ) < f ^ { L } ( \widehat { x } , \alpha ) } \\
{ f ^ { R } ( x , \alpha ) \leq f ^ { R } ( \widehat { x } , \alpha ) }
\end{array} \text { or } \left\{\begin{array}{l}
f^{L}(x, \alpha) \leq f^{L}(\widehat{x}, \alpha) \\
f^{R}(x, \alpha)<f^{R}(\widehat{x}, \alpha)
\end{array}\right.\right. \\
& \text { or }\left\{\begin{array}{l}
f^{L}(x, \alpha)<f^{L}(\widehat{x}, \alpha) \\
f^{R}(x, \alpha)<f^{R}(\widehat{x}, \alpha)
\end{array} \text { for all } \alpha \in[0,1] .\right.
\end{aligned}
$$

Remark 29 (Wu 2008) Note that any nondominated solution of the problem (FOP) is its weakly nondominated solution.

Then, using a suitable ordering of the intervals $\widetilde{f}_{\alpha}(x)=\left[f^{L}(x, \alpha), f^{R}(x, \alpha)\right]$ for each $\alpha \in[0,1]$, the minimization of a fuzzy function over a feasible set $D$ can be transformed into a bi-objective optimization problem. Therefore, for the considered constrained optimization problem (FOP) with the fuzzy-valued objective function, we define the family of its associated nondifferentiable bi-objective optimization problems defined for each $\alpha \in[0,1]$ as follows

$$
\begin{gathered}
\left(f^{L}(x, \alpha), f^{R}(x, \alpha)\right) \rightarrow \min \quad\left(\mathrm{VP}_{\alpha}\right) \\
x \in D .
\end{gathered}
$$

For such a vector optimization problem, we define its (weak) Pareto solution in the following sense:

Definition 30 It is said that $\widehat{x} \in D$ is a weak Pareto solution of the bi-objective optimization problem $\left(\mathrm{VP}_{\alpha}\right)$ for some $\alpha \in[0,1]$ if there does not exist other $x \in D$ such that

$$
\left\{\begin{array}{l}
f^{L}(x, \alpha)<f^{L}(\widehat{x}, \alpha) \\
f^{R}(x, \alpha)<f^{R}(\widehat{x}, \alpha)
\end{array} .\right.
$$

Definition 31 It is said that $\widehat{x} \in D$ is a Pareto solution of the bi-objective vector optimization problem $\left(\mathrm{VP}_{\alpha}\right)$ for some $\alpha \in[0,1]$ if there does not exist other $x \in D$ such that

$$
\begin{aligned}
& \left\{\begin{array} { l } 
{ f ^ { L } ( x , \alpha ) < f ^ { L } ( \widehat { x } , \alpha ) } \\
{ f ^ { R } ( x , \alpha ) \leq f ^ { R } ( \widehat { x } , \alpha ) }
\end{array} \text { or } \left\{\begin{array}{l}
f^{L}(x, \alpha) \leq f^{L}(\widehat{x}, \alpha) \\
f^{R}(x, \alpha)<f^{R}(\widehat{x}, \alpha)
\end{array}\right.\right. \\
& \text { or }\left\{\begin{array}{l}
f^{L}(x, \alpha)<f^{L}(\widehat{x}, \alpha) \\
f^{R}(x, \alpha)<f^{R}(\widehat{x}, \alpha) .
\end{array}\right.
\end{aligned}
$$

The next results allows to tie the considered fuzzy optimization problem (FOP) and its associated bi-objective optimization problem $\left(\mathrm{VP}_{\alpha}\right)$. 
Proposition 32 Let $\widehat{x} \in D$ be a weakly nondominated solution (a nondominated solution) of the considered fuzzy optimization problem (FOP). Then, it is also a weak Pareto solution of the bi-objective optimization problem $\left(V P_{\alpha}\right)$ for any $\alpha \in[0,1]$.

The following results show the connections between the sets of solutions for the considered fuzzy optimization problem (FOP) and its associated bi-objective vector optimization problem $\left(\mathrm{VP}_{\alpha}\right)$

Proposition 33 If $\widehat{x} \in D$ is a Pareto solution of any bi-objective optimization problem $\left(V P_{\alpha}\right)$ for each $\alpha \in[0,1]$, then $\widehat{x}$ is also a nondominated solution of the considered fuzzy optimization problem (FOP).

Proposition 34 If $\widehat{x} \in D$ is a Pareto solution of the bi-objective vector optimization problem $\left(V P_{\widehat{\alpha}}\right)$ for some $\widehat{\alpha} \in[0,1]$, then $\widehat{x}$ is also a weakly nondominated solution of the considered fuzzy optimization problem (FOP).

However, if we use this approach to solve the considered optimization problem (FOP) with fuzzy-valued objective function, then we must take into account the fact that its optimal solution can be ambiguous. This is a consequence of the fact that, in general, there is not a unique Pareto solution of the associated the bi-objective optimization problem $\left(\mathrm{VP}_{\alpha}\right)$. Indeed, in the general case, Pareto solutions of the associated bi-objective optimization problem $\left(\mathrm{VP}_{\alpha}\right)$ form the set. However, there are methods for solving such a nonlinear optimization problem in which each its Pareto solution can be found as a minimizer of an extremum problem constructed in such approaches.

One of such approaches is a scalarization method. It is well-known (see, for example, Miettinen et al. 2004) that the vector optimization problem $\left(\mathrm{VP}_{\alpha}\right)$ can be solved by using scalarization method. In this approach, for the nondifferentiable bi-objective optimization problem $\left(\mathrm{VP}_{\alpha}\right)$, we construct the following scalarized optimization problem defined by

$$
\begin{gathered}
\lambda(\alpha) f^{L}(x, \alpha)+(1-\lambda(\alpha)) f^{R}(x, \alpha) \rightarrow \min \quad\left(\mathrm{P}_{\alpha}(\lambda)\right) \\
x \in D,
\end{gathered}
$$

where $\lambda(\alpha) \in[0,1]$.

Now, we show the connection between the sets of Pareto solutions of the nondifferentiable bi-objective optimization problem $\left(\mathrm{VP}_{\alpha}\right)$ and the set of minimizers of its scalarized optimization problem $\left(\mathrm{P}_{\alpha}(\lambda)\right)$. The first result says that any Pareto solution of the nondifferentiable bi-objective optimization problem $\left(\mathrm{VP}_{\alpha}\right)$ with a fixed $\alpha$-cut is also a minimizer in the scalarized optimization problem $\left(\mathrm{P}_{\alpha}(\lambda)\right)$ if the functions $f^{L}(\cdot, \alpha)$ and $f^{R}(\cdot, \alpha)$ are invex on $D$ with respect to the same function $\eta$.

Proposition 35 Let $\widehat{x} \in D$ be a (weakly) Pareto solution of the nondifferentiable bi-objective optimization problem $\left(V P_{\alpha}\right)$ with fixed $\alpha$-cut. Further, assume that the functions $f^{L}(\cdot, \alpha)$ and $f^{R}(\cdot, \alpha)$ for each $\alpha \in[0,1]$ are invex at $\widehat{x}$ on $D$ with respect to the same function $\eta$ and also constraint functions satisfy appropriate invexity assumptions at $\widehat{x}$ with respect to the same $\eta$. Then, there exists $\widehat{\lambda} \in(0,1)$ such that $\widehat{x}$ is a minimizer of the scalarized optimization problem $\left(P_{\alpha}(\widehat{\lambda})\right)$. 
Now, for any fixed $\alpha \in[0,1]$, we give the converse result to that formulated in Proposition 35.

Proposition 36 Let $\widehat{x} \in D$ be a minimizer of the the scalarized optimization problem $\left(P_{\alpha}(\widehat{\lambda})\right)$ for a fixed $\alpha \in[0,1]$.

(i) If $\widehat{\lambda}(\alpha) \in[0,1]$, then $\widehat{x}$ is a weak Pareto solution of the bi-objective nondifferentiable vector optimization problem $\left(V P_{\alpha}\right)$.

(ii) If $\widehat{\lambda}(\alpha) \in(0,1)$, then $\widehat{x}$ is a Pareto solution of the bi-objective nondifferentiable vector optimization problem $\left(V P_{\alpha}\right)$.

(iii) If $\widehat{\lambda}(\alpha) \in[0,1]$ and $\widehat{x}$ is a unique minimizer of $\left(P_{\alpha}(\widehat{\lambda})\right)$, then $\widehat{x}$ is a Pareto solution of the nondifferentiable bi-objective optimization problem $\left(V P_{\alpha}\right)$.

We now prove the Karush-Kuhn-Tucker optimality conditions for a weakly nondominated solution of the problem (FOP).

Theorem 37 Let $\widehat{x}$ be a feasible solution of the considered fuzzy optimization problem $(F O P)$. Further, assume that, for some $\widehat{\alpha} \in[0,1]$, there exist $\widehat{\lambda}(\widehat{\alpha}) \in(0,1), \widehat{\mu}(\widehat{\alpha}) \in$ $R^{m}, \widehat{\mu}(\widehat{\alpha}) \geq 0$ and $\widehat{\vartheta}(\widehat{\alpha}) \in R^{r}$ such that the following Karush-Kuhn-Tucker optimality conditions

$$
\begin{gathered}
0 \in \partial\left(\widehat{\lambda}(\widehat{\alpha}) f^{L}(\widehat{x}, \widehat{\alpha})+(1-\widehat{\lambda}(\widehat{\alpha})) f^{R}(\widehat{x}, \widehat{\alpha})\right) \\
+\sum_{j=1}^{m} \widehat{\mu}_{j}(\widehat{\alpha}) \partial g_{j}(\widehat{x})+\sum_{i=1}^{r} \widehat{\vartheta}_{i}(\widehat{\alpha}) \partial h_{i}(\widehat{x}), \\
\widehat{\mu}_{j}(\widehat{\alpha}) g_{j}(\widehat{x})=0, j \in J
\end{gathered}
$$

hold. If the left- and right-hand side functions $f^{L}(\cdot, \widehat{\alpha})$ and $f^{R}(\cdot, \widehat{\alpha})$ of the fuzzy objective function $\widetilde{f}_{\widehat{\alpha}}(\cdot)$ are invex at $\widehat{x}$ on $D$ with respect to $\eta$, the functions $g_{j}, j=1, \ldots, m$, $h_{i}, i \in I_{\alpha}^{+}(\widehat{x})=\left\{i \in I: \widehat{\vartheta}_{i}(\alpha)>0\right\}$, and $-h_{i}, i \in I_{\alpha}^{-}(\widehat{x})=\left\{i \in I: \widehat{\vartheta}_{i}(\alpha)<0\right\}$, are invex at $\widehat{x}$ on $D$ with respect to the same function $\eta$, then $\widehat{x}$ is a weakly nondominated of the considered fuzzy optimization problem (FOP).

Proof By assumption, $\widehat{x}$ is such a feasible solution of the considered fuzzy optimization problem (FOP) for which there exist $\widehat{\lambda}(\widehat{\alpha}) \in(0,1), \widehat{\mu}(\widehat{\alpha}) \in R^{m}, \widehat{\mu}(\widehat{\alpha}) \geq 0$ and $\widehat{\vartheta}(\alpha) \in R^{r}$ for some $\widehat{\alpha} \in[0,1]$ such that the Karush-Kuhn-Tucker optimality conditions (10) and (11) are fulfilled. By assumption, all functions involved in the problem (FOP) are locally Lipschitz. Therefore, all functions constituting the associated scalarized problem $\left(P_{\alpha}(\widehat{\lambda})\right)$ are also locally Lipschitz. Thus, (10) and (11) imply that $\widehat{x}$ is a Karush-Kuhn-Tucker point of the scalarized optimization problem $\left(P_{\widehat{\alpha}}(\widehat{\lambda})\right)$ for some $\widehat{\alpha} \in[0,1]$. By assumption, the left- and right-hand side functions $f^{L}(\cdot, \widehat{\alpha})$ and $f^{R}(\cdot, \widehat{\alpha})$ are invex at $\widehat{x}$ on $D$ with respect to $\eta$. Then the objective function in the associated scalarized optimization problem $\left(P_{\widehat{\alpha}}(\widehat{\lambda})\right)$ is also invex at $\widehat{x}$ on $D$ with respect to $\eta$. By assumption, also $g_{j}, j=1, \ldots, m, h_{i}, i \in I_{\alpha}^{+}(\widehat{x})=\left\{i \in I: \widehat{\vartheta}_{i}(\alpha)>0\right\}$, and $-h_{i}, i \in I_{\alpha}^{-}(\widehat{x})=\left\{i \in I: \widehat{\vartheta}_{i}(\alpha)<0\right\}$, are invex at $\widehat{x}$ on $D$ with respect to the same function $\eta$. Then, by Definition 25 and the definition of a locally Lipschitz invex 
crisp function (see Definition 15), the inequalities

$$
\begin{aligned}
& f^{L}(x, \widehat{\alpha})-f^{L}(\widehat{x}, \widehat{\alpha}) \geq\left\langle\xi^{L}, \eta(x, \widehat{x})\right\rangle, \forall \xi^{L} \in \partial f^{L}(\widehat{x}, \widehat{\alpha}), \\
& f^{R}(x, \widehat{\alpha})-f^{R}(\widehat{x}, \widehat{\alpha}) \geq\left\langle\xi^{R}, \eta(x, \widehat{x})\right\rangle, \forall \xi^{R} \in \partial f^{R}(\widehat{x}, \widehat{\alpha}), \\
& g_{j}(x)-g_{j}(\widehat{x}) \geq\left\langle\zeta_{j}, \eta(x, \widehat{x})\right\rangle, \forall \zeta_{j} \in \partial g_{j}(\widehat{x}), j=1, \ldots, m, \\
& h_{i}(x)-h_{i}(\widehat{x}) \geq\left\langle\zeta_{i}, \eta(x, \widehat{x})\right\rangle, \forall \varsigma_{i} \in \partial h_{i}(\widehat{x}), i \in I_{\alpha}^{+}(\widehat{x}), \\
& -h_{i}(x)+h_{i}(\widehat{x}) \geq\left\langle-\varsigma_{i}, \eta(x, \widehat{x})\right\rangle, \forall\left(-\varsigma_{i}\right) \in \partial\left(-h_{i}(\widehat{x})\right), i \in I_{\alpha}^{-}(\widehat{x})
\end{aligned}
$$

hold for all $x \in D$. Multiplying (12) and (13) by $\widehat{\lambda}(\widehat{\alpha})$ and $1-\widehat{\lambda}(\widehat{\alpha})$, respectively, each inequality (14) by $\widehat{\mu}_{j}(\widehat{\alpha}), j \in J$, each inequality (15) by $\widehat{\vartheta}_{i}(\widehat{\alpha}), i \in I_{\alpha}^{+}(\widehat{x})$, each inequality (16) by $-\widehat{\vartheta}_{j}(\widehat{\alpha}), i \in I_{\alpha}^{-}(\widehat{x})$, and then adding the resulting inequalities, we get, for all $x \in D$,

$$
\begin{aligned}
& \widehat{\lambda}(\widehat{\alpha}) f^{L}(x, \widehat{\alpha})+(1-\widehat{\lambda}(\widehat{\alpha})) f^{R}(x, \widehat{\alpha})+\sum_{j=1}^{m} \widehat{\mu}_{j}(\widehat{\alpha}) g_{j}(x)+\sum_{i=1}^{r} \widehat{\vartheta}_{i}(\widehat{\alpha}) h_{i}(x) \\
& \quad-\left(\widehat{\lambda}(\widehat{\alpha}) f^{L}(\widehat{x}, \widehat{\alpha})+(1-\widehat{\lambda}(\widehat{\alpha})) f^{R}(\widehat{x}, \widehat{\alpha})+\sum_{j=1}^{m} \widehat{\mu}_{j}(\widehat{\alpha}) g_{j}(\widehat{x})+\sum_{i=1}^{r} \widehat{\vartheta}_{i}(\widehat{\alpha}) h_{i}(\widehat{x})\right) \\
& \quad \geq\left\langle\widehat{\lambda}(\widehat{\alpha}) \xi^{L}+(1-\widehat{\lambda}(\widehat{\alpha})) \xi^{R}+\sum_{j=1}^{m} \widehat{\mu}_{j}(\widehat{\alpha}) \zeta_{j}+\sum_{i=1}^{r} \widehat{\vartheta}_{i}(\widehat{\alpha}) \varsigma_{i}, \eta(x, \widehat{x})\right\rangle .
\end{aligned}
$$

Then, by the Karush-Kuhn-Tucker optimality condition (10), the relation above implies that the inequality

$$
\begin{aligned}
\widehat{\lambda}(\widehat{\alpha}) f^{L}(x, \widehat{\alpha})+(1-\widehat{\lambda}(\widehat{\alpha})) f^{R}(x, \widehat{\alpha})+\sum_{j=1}^{m} \widehat{\mu}_{j}(\widehat{\alpha}) g_{j}(x)+\sum_{i=1}^{r} \widehat{\vartheta}_{i}(\widehat{\alpha}) h_{i}(x) \\
\geq \widehat{\lambda}(\widehat{\alpha}) f^{L}(\widehat{x}, \widehat{\alpha})+(1-\widehat{\lambda}(\widehat{\alpha})) f^{R}(\widehat{x}, \widehat{\alpha}) \\
\quad+\sum_{j=1}^{m} \widehat{\mu}_{j}(\widehat{\alpha}) g_{j}(\widehat{x})+\sum_{i=1}^{r} \widehat{\vartheta}_{i}(\widehat{\alpha}) h_{i}(\widehat{x})
\end{aligned}
$$

holds for all $x \in D$. Using $x \in D, \widehat{x} \in D, \widehat{\mu}_{j} \geq 0, j \in J$, together with the Karush-Kuhn-Tucker optimality condition (11), we get that the inequality

$$
\begin{gathered}
\widehat{\lambda}(\widehat{\alpha}) f^{L}(x, \widehat{\alpha})+(1-\widehat{\lambda}(\widehat{\alpha})) f^{R}(x, \widehat{\alpha}) \geq \\
\widehat{\lambda}(\widehat{\alpha}) f^{L}(\widehat{x}, \widehat{\alpha})+(1-\widehat{\lambda}(\widehat{\alpha})) f^{R}(\widehat{x}, \widehat{\alpha})
\end{gathered}
$$

holds for all $x \in D$ and for some $\widehat{\alpha} \in[0,1]$. Since the set of all feasible solutions in the scalarized optimization problem $\left(P_{\widehat{\alpha}}(\widehat{\lambda})\right)$ is the same as in the problem (FOP), (17) implies that $\widehat{x}$ is a minimizer of the problem $\left(P_{\widehat{\alpha}}(\widehat{\lambda})\right)$. The scalarized optimization 
problem $\left(P_{\widehat{\alpha}}(\widehat{\lambda})\right)$ is the weighting optimization problem associated to the nondifferentiable bi-objective optimization problem $\left(\mathrm{VP}_{\widehat{\alpha}}\right)$. Further, by assumption, its weights are strictly positive real numbers. Hence, by Proposition 34 ii), it follows that $\widehat{x}$ is a Pareto solution for the nondifferentiable bi-objective optimization problem $\left(\mathrm{VP}_{\widehat{\alpha}}\right)$. Then, by Proposition 34, $\widehat{x}$ is a weakly nondominated solution of the considered fuzzy optimization problem (FOP). Thus, the proof of this theorem is completed.

Next, we present the Karush-Kuhn-Tucker optimality conditions for a nondominated solution of the considered fuzzy optimization problem (FOP).

Theorem 38 Let $\widehat{x}$ be a feasible solution of the considered fuzzy optimization problem $(F O P)$. Further, assume that there exist $\widehat{\lambda}(\alpha) \in(0,1), \widehat{\mu}(\alpha) \in R^{m}, \widehat{\mu}(\alpha) \geq 0$ and $\widehat{\vartheta}(\alpha) \in R^{r}$ such that the following Karush-Kuhn-Tucker optimality conditions

$$
\begin{aligned}
& 0 \in \partial\left(\widehat{\lambda}(\alpha) f^{L}(\widehat{x}, \alpha)+(1-\widehat{\lambda}(\alpha)) f^{R}(\widehat{x}, \alpha)\right) \\
& \quad+\sum_{j=1}^{m} \widehat{\mu}_{j}(\alpha) \partial g_{j}(\widehat{x})+\sum_{i=1}^{r} \widehat{\vartheta}_{i}(\alpha) \partial h_{i}(\widehat{x}), \\
& \widehat{\mu}_{j}(\alpha) g_{j}(\widehat{x})=0, j \in J
\end{aligned}
$$

hold for each $\alpha \in[0,1]$. If the fuzzy objective function $\widetilde{f}$ is an invex fuzzy function at $\widehat{x}$ on $D$ with respect to $\eta$, the functions $g_{j}, j=1, \ldots, m, h_{i}, i \in I_{\alpha}^{+}(\widehat{x})=$ $\left\{i \in I: \widehat{\vartheta}_{i}(\alpha)>0\right\}$, and $-h_{i}, i \in I_{\alpha}^{-}(\widehat{x})=\left\{i \in I: \widehat{\vartheta}_{i}(\alpha)<0\right\}$, are invex at $\widehat{x}$ on $D$ with respect to the same function $\eta$, then $\widehat{x}$ is a nondominated solution of the considered fuzzy optimization problem (FOP).

Proof By assumption, $\widehat{x}$ is such a feasible solution of the considered fuzzy optimization problem (FOP) for which there exist $\widehat{\lambda}(\alpha) \in(0,1), \widehat{\mu}(\alpha) \in R^{m}, \widehat{\mu}(\alpha) \geq 0$ and $\widehat{\vartheta}(\alpha) \in R^{r}$ such that the Karush-Kuhn-Tucker optimality conditions (18) and (19) are fulfilled. Since the functions involved in the problem (FOP) are locally Lipschitz, the functions constituting the associated scalarized problem $\left(\mathrm{P}_{\alpha}(\widehat{\lambda})\right)$ are also locally Lipschitz. Hence, by (18) and (19), it follows that $\widehat{x}$ is a Karush-Kuhn-Tucker point of the scalarized optimization problem $\left(\mathrm{P}_{\alpha}(\widehat{\lambda})\right)$ for each $\alpha \in[0,1]$. By assumption, the fuzzy objective function $\tilde{f}$ is an invex fuzzy function at $\widehat{x}$ on $D$ with respect to $\eta$. Then the objective function in the associated scalarized optimization problem $\left(\mathrm{P}_{\alpha}(\widehat{\lambda})\right)$ is also invex at $\widehat{x}$ on $D$ with respect to the same function $\eta$. By assumption, also the constraint functions $g_{j}, j=1, \ldots, m, h_{i}, i \in I_{\alpha}^{+}(\widehat{x})=\left\{i \in I: \widehat{\vartheta}_{i}(\alpha)>0\right\}$, and the functions $-h_{i}, i \in I_{\alpha}^{-}(\widehat{x})=\left\{i \in I: \widehat{\vartheta}_{i}(\alpha)<0\right\}$, are invex at $\widehat{x}$ on $D$ with respect to the same function $\eta$. This means that all functions constituting the scalarized optimization problem $\left(\mathrm{P}_{\alpha}(\widehat{\lambda})\right)$ are invex at $\widehat{x}$ on $D$ with respect to the same function $\eta$. Then, in the similar way as in the proof of Theorem 37 , it can be established that $\widehat{x}$ is a minimizer of the associated scalarized optimization problem $\left(\mathrm{P}_{\alpha}(\widehat{\lambda})\right)$ for each $\alpha \in[0,1]$. Since the scalarized optimization problem $\left(\mathrm{P}_{\alpha}(\widehat{\lambda})\right)$ is the weighting optimization problem associated to the considered fuzzy optimization problem (FOP) and, by assumption, its weights are strictly positive real numbers, therefore, by Proposition 36 ii), it follows that $\widehat{x}$ is a Pareto solution for the nondifferentiable bi-objective optimization problem 
$\left(\mathrm{VP}_{\alpha}\right)$ for each $\alpha \in[0,1]$. Hence, by Proposition $33, \widehat{x}$ is a nondominated solution of the considered fuzzy optimization problem (FOP). Then, the proof of this theorem is completed.

Now, we prove the Karush-Kuhn-Tucker necessary optimality conditions for a feasible solution $\widehat{x}$ to be a weakly nondominated solution of the considered constrained optimization problem (FOP) with fuzzy-valued objective function.

Theorem 39 Let $\widehat{x} \in D$ be a weakly nondominated solution of the considered fuzzy optimization problem (FOP). Further, assume $\widetilde{f}$ is a fuzzy invex function at $\widehat{x}$ on $D$ with respect to $\eta$, and also constraint functions satisfy appropriate invexity assumptions at $\widehat{x}$ with respect to the same function $\eta$ and, moreover, the Slater constraint qualification is satisfied at $\widehat{x}$ for $(F O P)$. Then, there exist $\widehat{\alpha} \in[0,1], \widehat{\lambda}(\alpha) \in[0,1], \widehat{\mu}(\widehat{\alpha}) \in R^{m}$, $\widehat{\mu}(\widehat{\alpha}) \geq 0$ and $\widehat{\vartheta}(\widehat{\alpha}) \in R^{r}$ such that the Karush-Kuhn-Tucker optimality conditions

$$
\begin{gathered}
0 \in \partial\left(\widehat{\lambda}(\alpha) f^{L}(\widehat{x}, \widehat{\alpha})+(1-\widehat{\lambda}(\alpha)) f^{R}(\widehat{x}, \widehat{\alpha})\right) \\
+\sum_{j=1}^{m} \widehat{\mu}_{j}(\widehat{\alpha}) \partial g_{j}(\widehat{x})+\sum_{i=1}^{r} \widehat{\vartheta}_{i}(\widehat{\alpha}) \partial h_{i}(\widehat{x}), \\
\widehat{\mu}_{j}(\alpha) g_{j}(\widehat{x})=0, j \in J
\end{gathered}
$$

hold.

Proof Assume that $\hat{x}$ is a weakly nondominated solution of the considered fuzzy optimization problem (FOP). Hence, by Proposition 32, there exists an $\widehat{\alpha}$-cut such that $\widehat{x}$ is a weak Pareto solution of the bi-objective vector optimization problem $\left(\mathrm{VP}_{\widehat{\alpha}}\right)$ associated with the problem (FOP). By Proposition 35, it follows that $\widehat{x}$ is a minimizer of of the scalarized optimization problem $\left(\mathrm{P}_{\widehat{\alpha}} \widehat{\lambda}\right)$ ) for a fixed $\widehat{\alpha} \in[0,1]$. Hence, by Lagrange Multiplier Rule (see Theorem 6.1.1 Clarke 1983), there exist Lagrange multipliers $\widehat{\theta}(\widehat{\alpha}) \in R_{+}, \widehat{\mu}(\widehat{\alpha}) \in R_{+}^{m}, \widehat{\vartheta}(\widehat{\alpha}) \in R^{r}$, not all zero, and $\widehat{\lambda}(\widehat{\alpha}) \in[0,1]$ such that

$$
\begin{gathered}
0 \in \widehat{\theta}(\widehat{\alpha}) \partial\left(\widehat{\lambda}(\widehat{\alpha}) f^{L}(\widehat{x}, \widehat{\alpha})+(1-\widehat{\lambda}(\widehat{\alpha})) f^{R}(\widehat{x}, \widehat{\alpha})\right) \\
+\sum_{j=1}^{m} \widehat{\mu}_{j}(\widehat{\alpha}) \partial g_{j}(\widehat{x})+\sum_{i=1}^{r} \widehat{\vartheta}_{i}(\widehat{\alpha}) \partial h_{i}(\widehat{x}), \\
\widehat{\mu}_{j}(\widehat{\alpha}) g_{j}(\widehat{x})=0, j \in J .
\end{gathered}
$$

Since the Slater constraint qualification is satisfied at $\widehat{x}$ for the problem (FOP), Lagrange multiplier $\widehat{\theta}(\widehat{\alpha})$ can be set as equal to 1 in (22). Hence, (22) implies (20). This completes the proof of this theorem.

In the next corollary, we give the "separated" version of the Karush-Kuhn-Tucker optimality conditions which is, in general, weaker than those presented in the above theorem. 
Corollary 40 Let $\widehat{x} \in D$ be a weakly nondominated solution of the considered fuzzy optimization problem (FOP) and all hypotheses of Theorem 39 be fulfilled. Then, there exist $\widehat{\alpha} \in[0,1], \widehat{\lambda}(\widehat{\alpha}) \in[0,1], \widehat{\mu}(\widehat{\alpha}) \in R^{m}, \widehat{\mu}(\widehat{\alpha}) \geq 0$ and $\widehat{\vartheta}(\widehat{\alpha}) \in R^{r}$ such that

$$
\begin{aligned}
0 \in & \widehat{\lambda}(\widehat{\alpha}) \partial f^{L}(\widehat{x}, \widehat{\alpha})+(1-\widehat{\lambda}(\widehat{\alpha})) \partial f^{R}(\widehat{x}, \widehat{\alpha}) \\
& +\sum_{j=1}^{m} \widehat{\mu}_{j}(\widehat{\alpha}) \partial g_{j}(\widehat{x})+\sum_{i=1}^{r} \widehat{\vartheta}_{i}(\widehat{\alpha}) \partial h_{i}(\widehat{x}) .
\end{aligned}
$$

Proof Since all hypotheses of Theorem 39 are fulfilled, there exist $\widehat{\alpha} \in[0,1]$, $\widehat{\theta}(\widehat{\alpha}) \in R_{+}, \widehat{\mu}(\widehat{\alpha}) \in R_{+}^{m}, \widehat{\vartheta}(\widehat{\alpha}) \in R^{r}$, not all zero, and $\widehat{\lambda}(\widehat{\alpha}) \in[0,1]$ such that the Karush-Kuhn-Tucker necessary optimality conditions (20)-(21) are satisfied. Then, since $\widehat{\lambda}(\widehat{\alpha})$ and $1-\widehat{\lambda}(\widehat{\alpha})$ are nonnegative, by Corollary 13, the Karush-Kuhn-Tucker necessary optimality condition (20) implies the Karush-Kuhn-Tucker necessary optimality condition (23). This completes the proof of this corollary.

In order to illustrate the optimality results established in the paper, we give the example of a nondifferentiable optimization problem with fuzzy-valued objective function.

Example 41 Consider the following nondifferentiable nonconvex optimization problem with the fuzzy-valued objective function:

$$
\begin{gathered}
\tilde{f}(x)=\widetilde{2} \ln \left(\left(x^{2}+|x|+1\right) e\right) \ominus_{H} \tilde{1} \rightarrow \min \\
g_{1}(x)=x^{2}-5 x \leq 0,
\end{gathered}
$$

where $\tilde{1}$ and $\widetilde{2}$ are continuous triangular fuzzy numbers which are defined as triples $\widetilde{1}=(0,1,2)$ and $\widetilde{2}=(0,2,4)$. Then, by $(5)$, the $\alpha$-level sets of these triangular fuzzy numbers are $\widetilde{1}_{\alpha}=[\alpha, 2-\alpha]$ and $\widetilde{2}_{\alpha}=[2 \alpha, 4-2 \alpha]$, respectively. Note that the set of all feasible solutions of (FOP1) is $D=\left\{x \in R: x^{2}-5 x \leq 0\right\}=[0,5]$ and $\widehat{x}=0$ is a feasible solution of (FOP1). Further, by (2) and (3), the $\alpha$-level cut of the fuzzy objective function is defined by

$$
\begin{aligned}
\tilde{f}_{\alpha}(x)= & {\left[2 \alpha \ln \left(\left(x^{2}+|x|+1\right) e\right)-\alpha\right.} \\
& \left.(4-2 \alpha) \ln \left(\left(x^{2}+|x|+1\right) e\right)+\alpha-2\right]
\end{aligned}
$$

for all $\alpha \in[0,1]$. For example, the $\alpha$-level cuts of the fuzzy objective function $\tilde{f}$ for $\alpha=0, \alpha=\frac{1}{2}$ and $\alpha=1$ are as follows (see also Figs. 1,2,3):

$$
\begin{gathered}
\tilde{f}_{\alpha=0}(x)=\left[0,4 \ln \left(\left(x^{2}+|x|+1\right) e\right)-2\right] \\
\widetilde{f}_{\alpha=\frac{1}{2}}(x)=\left[\ln \left(\left(x^{2}+|x|+1\right) e\right)-\frac{1}{2}, 3 \ln \left(\left(x^{2}+|x|+1\right) e\right)-\frac{3}{2}\right] \\
\widetilde{f}_{\alpha=1}(x)=\left[2 \ln \left(\left(x^{2}+|x|+1\right) e\right)-1,2 \ln \left(\left(x^{2}+|x|+1\right) e\right)-1\right]
\end{gathered}
$$




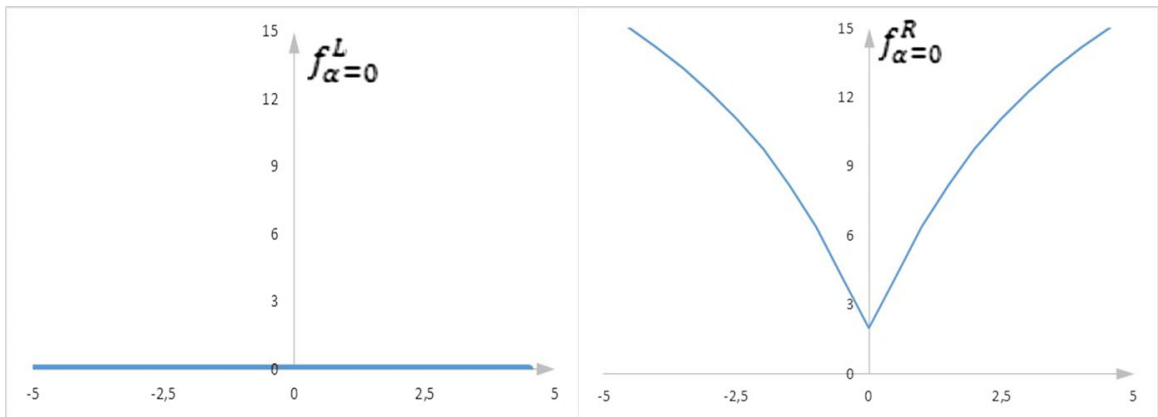

Fig. 1 Graphs of the left- and right-hand functions of $\widetilde{f}_{\alpha=0}$

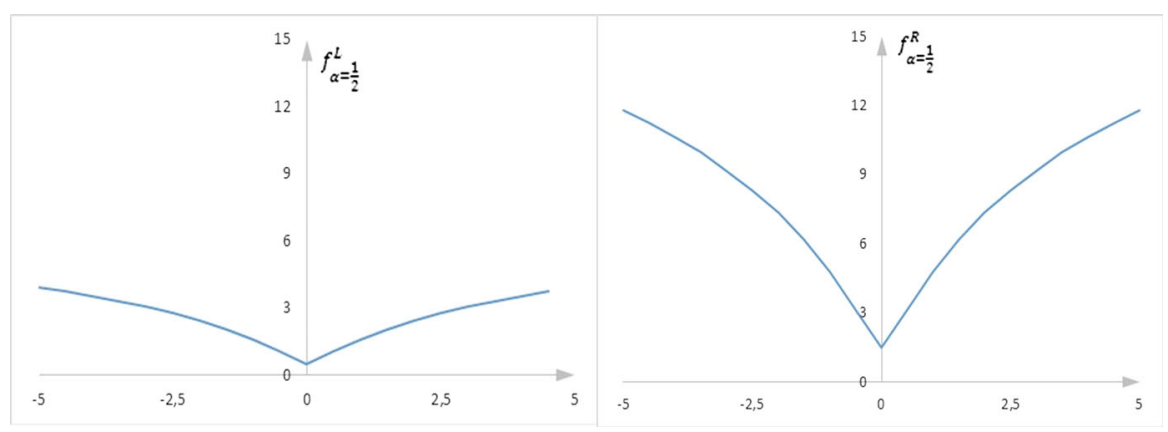

Fig. 2 Graphs of the left- and right-hand functions of $\widetilde{f}_{\alpha=\frac{1}{2}}$

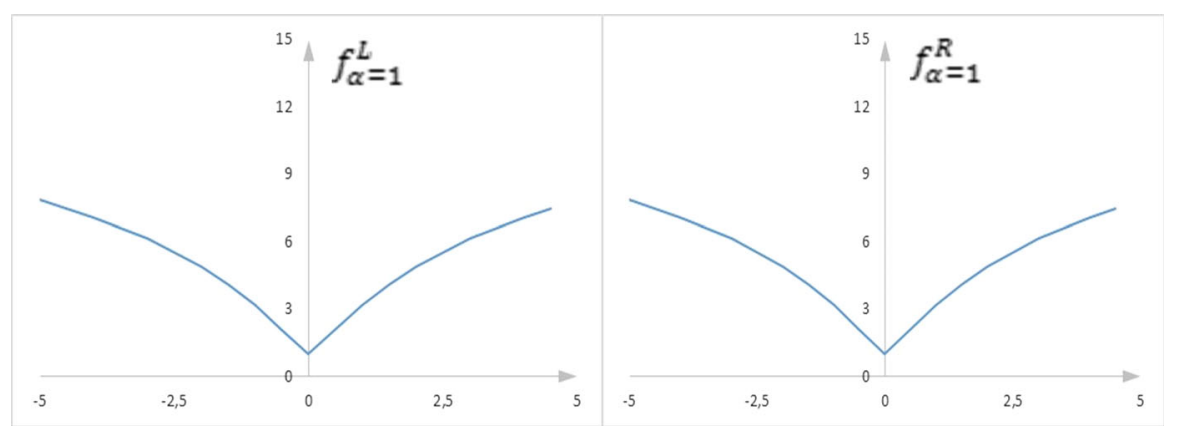

Fig. 3 Graphs of the left- and right-hand functions of $\tilde{f}_{\alpha=1}$

Clearly, the left- and right-hand side functions $f^{L}(\cdot, \alpha)$ and $f^{R}(\cdot, \alpha)$ are not convex and so $\tilde{f}$ is not convex (see also Figs. 1,2,3). Further, also note that $f^{L}(\cdot, \alpha)$ and $f^{R}(\cdot, \alpha)$ are not differentiable at $\widehat{x}=0$ and, therefore, $\tilde{f}$ is not level-wise differentiable at this point (see Definition 4.2 Wu (2007)). For these reasons, we are not able to find a (weakly) nondominated of the nondifferentiable fuzzy optimization problem (FOP1) by using the Karush-Kuhn-Tucker optimality conditions for differentiable fuzzy optimization problems (see, for example, Panigrahi et al. 2008; Pathak and Pirzada 2001; Ruziyeva and Dempe 2015; Wu 2007), Wu 2008). However, we 
show that the Karush-Kuhn-Tucker optimality conditions established in the present paper are applicable for the considered nondifferentiable fuzzy optimization problem (FOP1). Indeed, the Karush-Kuhn-Tucker optimality conditions (18) and (19) are fulfilled with Lagrange multipliers $\widehat{\lambda}(\alpha)=\frac{1}{4}$ and $\widehat{\mu}_{1}(\alpha)=1$ for each $\alpha \in[0,1]$. Note that all functions constituting (FOP1) are locally Lipschitz, that is, the objective function is a locally Lipschitz fuzzy function in the context of Definition 19. Further, we show that the functions constituting the nondifferentiable fuzzy optimization problem (FOP1) satisfy invexity hypotheses of Theorem 38. In order to do this, let us define $\eta: D \times D \rightarrow R$ by $\eta(x, \widehat{x})=\ln \left(x^{2}+|x|+1\right)-\ln \left(\widehat{x}^{2}+|\widehat{x}|+1\right)$. Hence, the functions $f^{L}(\cdot, \alpha)$ and $f^{R}(\cdot, \alpha)$ are invex at $\widehat{x}=0$ on $D$. Indeed, note that inequalities (8) and (9) are fulfilled at $\widehat{x}=0$ for all $x \in D$ with respect to $\eta$ defined above. Then, by Definition 25 , the fuzzy objective function $\widetilde{f}$ is invex at $\widehat{x}=0$ on $D$ with respect to $\eta$. Note that the constraint function $g_{1}$ is also invex at $\widehat{x}=0$ on $D$ with respect to the same function $\eta$ as it follows by Definition 15. Since all hypotheses of Theorem 38 are fulfilled, $\widehat{x}=0$ is a nondominated solution of the problem (FOP1).

\section{Conclusions}

In the paper, the definition of Clarke generalized derivative and Clarke generalized gradient have been defined for a nonsmooth fuzzy function and they have been used in defining the introduced concept of invexity for a nondifferentiable fuzzy mapping. Further, a nondifferentiable optimization problem with fuzzy objective function and with both inequality and equality constraints has been considered in which the involved functions are locally Lipschitz. The optimality conditions have been established for such a nonsmooth extremum problem. In proving this result, for the considered nondifferentiable fuzzy optimization problem, its associated bi-objective optimization problem has been constructed. It has been established the connection between Pareto solutions of this vector optimization problem and (weakly) nondominated solutions of the considered nondifferentiable fuzzy optimization problem. Furthermore, this biobjective optimization problem has been used in formulating Karush-Kuhn-Tucker optimality conditions for the original nonsmooth fuzzy optimization problem. Thus, the sufficient optimality conditions have been proved for the considered nondifferentiable optimization problem with fuzzy objective function under invexity hypotheses.

The open question is whether it is possible to prove similar optimality conditions for other classes of nondifferentiable fuzzy extremum problems. However, it seems that the techniques employed in this paper can be used in proving similar results for other classes of fuzzy mathematical programming problems with locally Lipschitz functions, including fuzzy decision making problems. We shall investigate these problems in the subsequent papers.

Open Access This article is licensed under a Creative Commons Attribution 4.0 International License, which permits use, sharing, adaptation, distribution and reproduction in any medium or format, as long as you give appropriate credit to the original author(s) and the source, provide a link to the Creative Commons licence, and indicate if changes were made. The images or other third party material in this article are included in the article's Creative Commons licence, unless indicated otherwise in a credit line to the material. If 
material is not included in the article's Creative Commons licence and your intended use is not permitted by statutory regulation or exceeds the permitted use, you will need to obtain permission directly from the copyright holder. To view a copy of this licence, visit http://creativecommons.org/licenses/by/4.0/.

\section{References}

Ammar, E. E., \& Metz, J. (1992). On fuzzy convexity and parametric fuzzy optimization. Fuzzy Sets and Systems, 49, 135-141.

Arana-Jiménez, M., Rufián-Lizana, A., Chalco-Cano, Y., \& Román-Flores, H. (2015). Generalized convexity in fuzzy vector optimization through a linear ordering. Information Sciences, 312, 13-24.

Behera, S. K., \& Nayak, J. R. (2014). Optimality criteria for fuzzy pseudo convex functions. Indian Journal of Science and Technology, 7, 986-990.

Chalco-Cano, Y., Lodwick, W. A., Osuna-Gomez, R., \& Rufian-Lizana, A. (2016). The Karush-KuhnTucker optimality conditions for fuzzy optimization problems. Fuzzy Optimization and Decision Making, 15, 57-73.

Clarke, F. H. (1983). Optimization and Nonsmooth Analysis. Wiley: A Wiley-Interscience Publication.

Miettinen, K. (2004). Nonlinear multiobjective optimization. International Series in Operations Research \& Management Science, Kluwer Academic Publishers, Massachusetts.

Mishra, S. K., Wang, S. Y., \& Lai, K. K. (2006). Explicitly B-preinvex fuzzy mappings. International Journal of Computer Mathematics, 83, 39-47.

Nanda, S., \& Kar, K. (1992). Convex fuzzy mappings. Fuzzy Sets and Systems, 48, 129-132.

Noor, M. A. (1994). Fuzzy preinvex functions. Fuzzy Sets and Systems, 79, 267-269.

Osuna-Gómez, R., Chalco-Cano, Y., Osuna-Gomez, R., \& Rufian-Lizana, A. (2016). Necessary and sufficient conditions for fuzzy optimality problems. Fuzzy Sets and Systems, 296, 112-123.

Panigrahi, M., Panda, G., \& Nanda, S. (2008). Convex fuzzy mapping with differentiability and its application in fuzzy optimization. European Journal of Operational Research, 185, 47-62.

Pathak, V.D., \& Pirzada, U.M. (2001). Necessary and sufficient optimality conditions for nonlinear fuzzy optimization problem. Sutra: International Journal of Mathematical Science Education, 4, 1-16

Reiland, T. W. (1990). Nonsmooth invexity. Bulletin of the Australian Mathematical Society, 42, 437-446.

Rufián-Lizana, A., Chalco-Cano, Y., Osuna-Gomez, R., \& Ruiz-Garzon, G. (2012). On invex fuzzy mappings and fuzzy variational-like inequalities. Fuzzy Sets and Systems, 200, 84-98.

Ruziyeva, A., \& Dempe, S. (2015). Optimality conditions in nondifferentiable fuzzy optimization. Optimization, 64, 349-363.

Syau, Y. R. (1999). Preinvex fuzzy mapping. Computers \& Mathematics with Applications, 37, 31-39.

Syau, Y. R. (2000). Invex and generalized convex fuzzy mappings. Fuzzy Sets and Systems, 115, 455-461.

Syau, Y. R. (2001). Generalization of preinvex and B-vex fuzzy mappings. Fuzzy Sets and Systems, 120, 533-542.

Tanaka, H., Ukuda, T., \& Asal, K. (1984). On fuzzy mathematical programming. Journal of Cybernetics, 3, 37-46.

Wang, C. X., \& Wu, C. X. (2003). Derivatives and subdifferential of convex fuzzy mappings and application to convex fuzzy programming. Fuzzy Sets and Systems, 138, 559-591.

Wu, H.-Ch. (2007). The Karush-Kuhn-Tucker optimality conditions for the optimization problem with fuzzy-valued objective function. Mathematical Methods of Operations Research, 66, 203-224.

Wu, H.-Ch. (2008). The optimality conditions for optimization problems with fuzzy-valued objective functions. Optimization, 57, 473-489.

Wu, H.-Ch. (2009). The Karush-Kuhn-Tucker optimality conditions for multi-objective programming problems with fuzzy-valued objective functions. Fuzzy Optimization and Decision Making, 8, 1-28.

Yan, H., \& Xu, J. (2002). A class of convex fuzzy mappings. Fuzzy Sets and Systems, 129, 47-56.

Publisher's Note Springer Nature remains neutral with regard to jurisdictional claims in published maps and institutional affiliations. 